\title{
Microscale Tracking of Surgical Instrument Motion
}

\author{
Cameron N. Riviere and Pradeep K. Khosla \\ Center for Medical Robotics and Computer Assisted Surgery \\ The Robotics Institute, Carnegie Mellon University, Pittsburgh, PA \\ $\{$ camr,pkk\}@ri.cmu.edu \\ www.mrcas.ri.cmu.edu
}

\begin{abstract}
An apparatus for accurate three-dimensional tracking of the tip of a microsurgical instrument has been developed for laboratory use. The system is useful for evaluation of microsurgical instrument designs and devices for accuracy enhancement (both robotic devices and active hand-held instruments), as well as for assessment and training of microsurgeons. It can also be used as a high-precision input interface to microsurgical simulators. The system involves illumination of the workspace and optical sensing of the position of a small reflective ball at the instrument tip, and therefore requires no wiring connection to the instrument being tracked. Sensing is performed by two position-sensitive photodiodes, placed orthogonally. The rms noise per coordinate is presently $7 \mathrm{mi}-$ crons. Preliminary results are presented. The photodiodes exhibit some degree of nonlinearity, which can be calibrated. The goal is to achieve an rms noise level of 1 micron. This is expected to be attainable via a synchronous detection scheme which has not yet been implemented.
\end{abstract}

\section{Introduction}

The need for high accuracy is intrinsic to microsurgery [1]. Vitreoretinal microsurgeons, unassisted by accuracy enhancement devices, are capable of accuracy to well below $100 \mu \mathrm{m}$ for brief periods of time [2]. Vitreoretinal microsurgery is among the most demanding of specialties in terms of positioning accuracy; there appears to be some consensus within the field on the goal of $10 \mu \mathrm{m}$ accuracy [3]. Thorough evaluation of microsurgical accuracy enhancement devices (whether robotic manipulators [4,5,6,7] or active hand-held instruments [8]), as well as more traditional passive instruments, therefore requires sensing of instrument tip position in three dimensions, and with accuracy to less than $10 \mu \mathrm{m}$. A sensing apparatus meeting these requirements would also be useful for assessment and training of surgeons, as well as comprehensive characterization of erroneous manual motion at the microscale (including non-tremorous components [2]), and could be used as a high-precision input interface for microsurgical simulators.

Ease of manipulation and avoidance of fatigue are important in microsurgery, and as a result, hand-held instruments are of course designed to be lightweight. In order to keep the sensing apparatus from altering the dynamics of the motion to 
be tracked, it is desirable to avoid configurations that require physical contact between sensor and instrument, or that require significant added mass to be attached to the instrument.

Numerous commercial systems are commonly used in tracking surgical instruments [9], including Optotrak systems (Northern Digital, Waterloo, Can.) [10], the miniBird (Ascension Technology Corp., Burlington, Vt.) [11], and Fastrak (Polhemus, Colchester, Vt.) [12]. These systems, as available off the shelf, offer high accuracy, but not high enough for the present application, and all require that a sensor module be attached to the instrument, usually resulting in a significant change in its mass. Another system, recently developed specifically for evaluating vitreoretinal surgical instruments and surgeons, is the Johns Hopkins MADSAM system [13], which utilizes Hall effect sensing. It provides accuracy to a few microns, and requires attaching only a magnet of $0.23 \mathrm{~g}$ mass to the instrument tip. The drawback of MADSAM is that it only tracks in one dimension. One technology that offers a promising alternative is the position-sensitive detector, or lateral-effect photodiode. A two-dimensional position-sensitive detector is an analog sensor that puts out currents related to the $x$ and $y$ position of a light spot on its active surface. Position-sensitive detectors are frequently used in remote position sensing applications $[14,15]$. They require no contact with the sensed object, and feature high position resolution and fast response [14], although they also often have significant thermal drift. Direct illumination from lasers or light-emitting diodes (LEDs) is typically used for sensing, but a reflective approach is also possible. The Apparatus to Sense Accuracy of Position (ASAP) has been developed for laboratory use, to meet the need for non-contact threedimensional tracking of the tip of a microsurgical instrument, without adding significant mass to the instrument, for applications in surgical performance evaluation, analysis, training, and simulation, and for testing of engineered devices for accuracy enhancement.

\section{Methods}

ASAP is a system for measurement of surgical instrument tip position in three dimensions. A hexagonal array of seven Hewlett-Packard HLMP-DG08 red (626 $\mathrm{nm}$ ) high-power light-emitting diodes (LEDs) illuminates the workspace. The LEDs are pulsed at $1 \mathrm{kHz}$ with $50 \%$ duty cycle. A white aluminum oxide ball $1.6 \mathrm{~mm}$ in diameter is affixed to the tip of a typical hand-held vitreoretinal microsurgical instrument. The instrument itself, and all other surfaces within view, are painted flat black. Reflected light from the ball is received by two photodiode sensors facing in orthogonal directions, each one oriented at 45 with respect to the LED array, providing sensing in three dimensions, with redundant measurement along the vertical axis. Each sensor is fitted with a red optical bandpass filter and a lens (Nikkor $50 \mathrm{~mm} \mathrm{1:2).} \mathrm{This} \mathrm{reflective} \mathrm{approach}$ obviates electrical connection to the instrument tip. Each sensor is mounted on a linear motion stage, and positioned roughly $10 \mathrm{~cm}$ from the back of the lens, putting it about $20 \mathrm{~cm}$ from the workspace. 
The photodiode sensors used in the system are pincushion-type positionsensitive detectors (PSDs) (S2044, Hamamatsu Corp., Bridgewater, N. J., USA). This type of sensor is a planar photodiode with an electrode at each of its four corners. Each electrode is connected to ground via a load resistor. A common fifth pin of the detector may be grounded, or biased by several volts. Incident light represents a photocurrent that then flows to ground via the load resistor, generating an analog signal. PSDs are characterized by high position resolution and fast response compared with other detectors such as charge-coupled devices. PSDs are quite susceptible to thermal drift; in order to avoid the effects of this, ASAP uses AC excitation of, and coupling to, the PSDs.

In the ASAP system, a $5.11 \mathrm{k} \Omega$ load is used for each signal, in parallel with a capacitance of $0.0068 \mu \mathrm{F}$. The voltage across the load is input to an instrumentation amplifier. The instrumentation amplifier output passes through a passive high-pass filter with $330 \mathrm{~Hz}$ cutoff. This signal is full-wave rectified and amplified, and then low-pass filtered with a $20 \mathrm{~Hz}$ second-order cutoff. This cutoff frequency is selected to minimize noise while allowing capture of the full bandwidth of hand motion, including the nominally 8-12 Hz physiological tremor band. The total gain of the circuit is $90 \mathrm{~dB}$.

A special-purpose analog-to-digital converter card for PSDs (DAS-PSP, Magen Scientific Corp., New York, N. Y., USA) is used to acquire the signals and calculate the position of the light spot on each photodiode [16]. The system samples data at $2 \mathrm{kHz}$, downsampling to $100 \mathrm{~Hz}$.

Looking into the front of the PSD, moving counterclockwise from the lower left, let $u_{1}, v_{1}, u_{2}$, and $v_{2}$, respectively, be the signals from the four electrodes. For the pincushion-type PSD, the coordinates ( $x$ and $z$ for the first PSD) of the light spot on the photodiode surface are calculated as follows [17]:

$$
\begin{aligned}
& x=L\left(\frac{\left(u_{2}+v_{1}\right)-\left(u_{1}+v_{2}\right)}{u_{1}+u_{2}+v_{1}+v_{2}}\right) \\
& z=L\left(\frac{\left(u_{2}+v_{2}\right)-\left(u_{1}+v_{1}\right)}{u_{1}+u_{2}+v_{1}+v_{2}}\right) .
\end{aligned}
$$

The second PSD likewise yields coordinates $y$ and $z$, respectively. The system is shown in Figure 1. Figure 2 displays ASAP with a mannequin face that has been prepared for realistic simulation of vitreoretinal microsurgery, including a sclerotomy to accommodate the surgical instrument.

\section{Results}

The root-mean-square noise in each of the three coordinate measurements is $7 \mu \mathrm{m}$. The position resolution of the system resulting from the discretization is approximately $0.2 \mu \mathrm{m}$. The linearity of the system is largely determined by the linearity of the PSDs. Hamamatsu reports typical nonlinearity of $\pm 40 \mu \mathrm{m}$ within a $0.9 \mathrm{~mm}$ radius of the center, and $\pm 70 \mu \mathrm{m}$ within a $4 \mathrm{~mm} \times 4 \mathrm{~mm}$ square, 


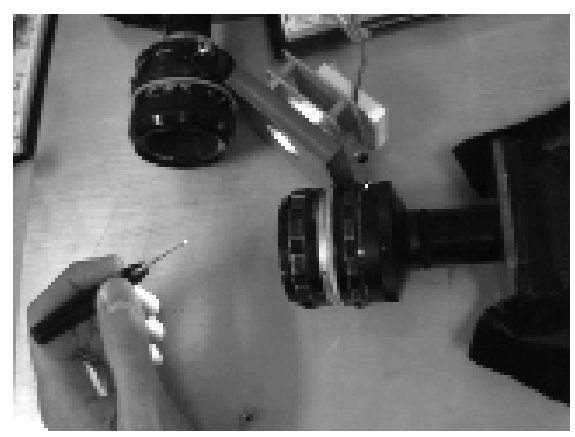

Fig. 1. The ASAP 3-D position measurement system for microsurgical evaluation. The LED array, lenses, and black instrument with white reflective ball are all visible.

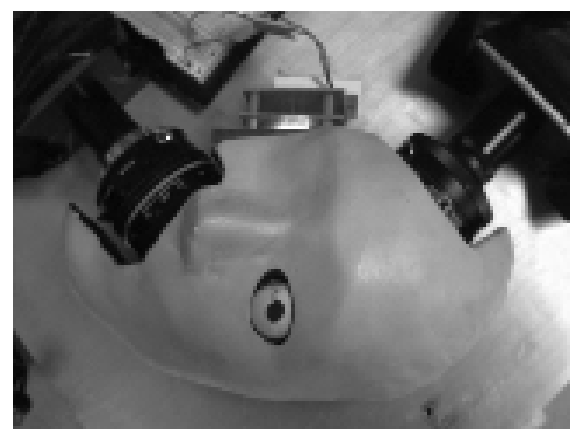

Fig. 2. The vitreoretinal microsurgery testbed constructed using ASAP for sensing within the simulated eye.

excluding the previous circular region. The workspace is a roughly cubical region with a volume of approximately $90 \mathrm{~mm}^{3}$.

Sample results from the ASAP system are displayed in Figures 3 through 5. Figure 3 presents a tracing of an orthogonal frame made with the instrument mounted on a manually operated 3-D linear stage. Nonlinear calibration has not yet been performed, and the nonlinearity in the system may be seen, e.g., in the departure of the frame's z-axis from the vertical. The figure also makes the repeatability of the system evident, as each of the three line segments in the figure is traced twice (one in each direction). Figure 4 represents a freehand attempt at a similar figure. Figure 5 presents a sample of data recorded while a subject holding the instrument attempted to keep it motionless, along with a recording of the system noise for comparison.

\section{Discussion}

To our knowledge, the data presented here represent the first recordings ever made of microsurgical instrument motion in three dimensions with this degree 


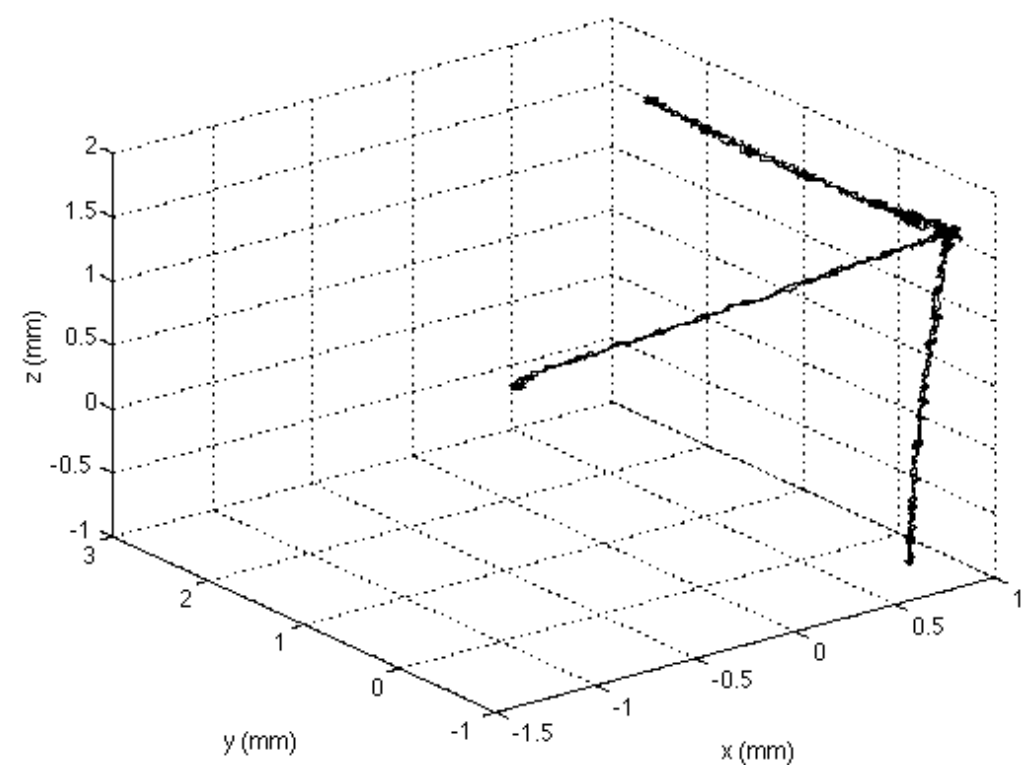

Fig. 3. Tracing of an orthogonal frame with surgical instrument mounted on a manual 3-D linear stage. In the course of the motion, each segment was traced twice (once in each direction).

of accuracy ( $7 \mu \mathrm{m}$ rms noise). The goal of the project is accuracy to $1 \mu \mathrm{m}$. This goal is expected to be attainable through the use of synchronous detection [15], which is the next step in the development of ASAP, and nonlinear calibration. The characteristic PSD nonlinearity evident in the system can be calibrated out, as the detectors are repeatable to submicron levels [14]. This work is in progress. As noted earlier, drift is already suppressed via AC excitation and coupling. The position detection error in ASAP, after nonlinear calibration, then consists largely of the noise.

Upon attaining the goal of $1 \mu \mathrm{m}$ accuracy, the system will be used in experimentation to obtain full 3-D quantification of instrument tip motion by vitreoretinal microsurgeons. These studies will lead to better understanding and modeling of involuntary and inadvertent components of the motion, especially non-tremorous components, which are little understood. This information is expected to enable the development of better algorithms and techniques for suppressing or compensating positioning error during microsurgery. In the future, ASAP will be applied also for evaluation of active devices for microsurgical accuracy enhancement, as well as new ergonomic designs for passive instruments.

The ASAP system described here can be duplicated at relatively low costless than US\$2000, excluding the cost of an IBM-compatible personal computer to operate the system. The system is intended for use in the laboratory, not in the operating room, where optical tracking of the instrument tip is problematic. 


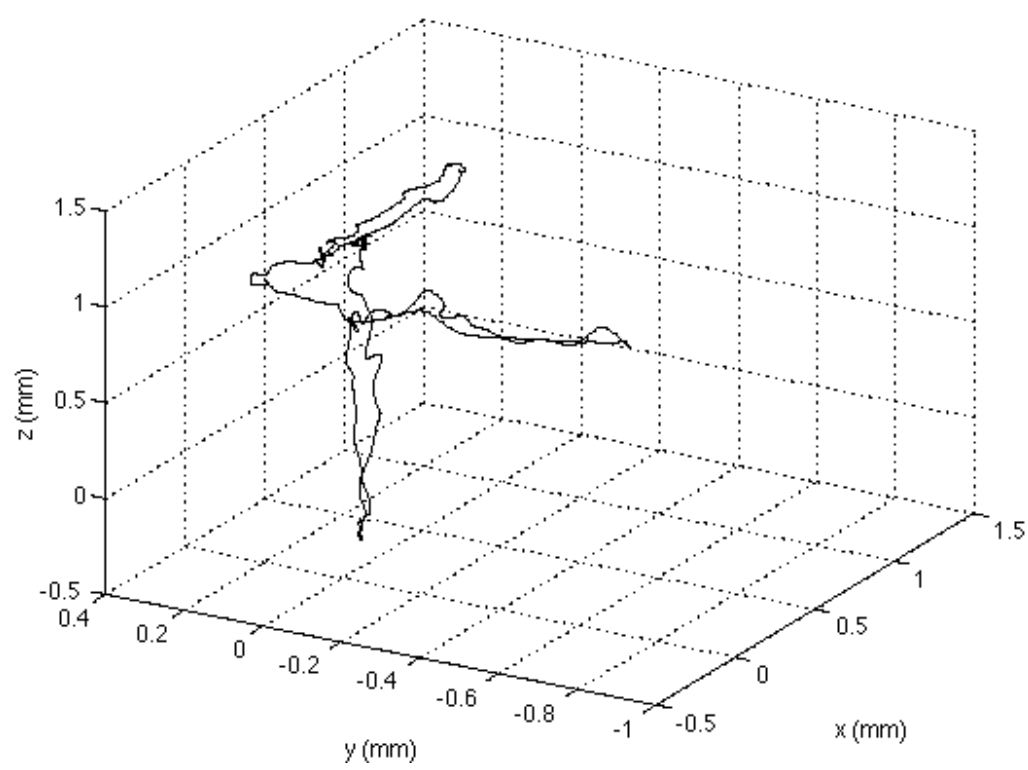

Fig. 4. Freehand tracing of an orthogonal frame. In the course of the motion, each segment was traced twice (once in each direction).

A separate effort in our laboratory involves inertial tracking of intraoperative instrument motion [18].

Plans for future work include not only further improving the accuracy, but also enlarging the workspace to approximately $1 \mathrm{~cm}^{3}$. Reducing the size of the marker ball would increase the workspace somewhat, but would also decrease the signal-to-noise ratio, as its decreased surface area would decrease the incident light to the sensors. Another possibility, conversion to a different PSD with larger active area, is presently being planned. The new PSDs will be from the DL series of UDT Sensors, Inc. (Hawthorne, Ca.), which offers superior linearity [14]. Readjustment of the optics may also be necessary.

\section{Conclusion}

A laboratory system for three-dimensional non-contact tracking of microsurgical instrument tip position has been developed. Optical tracking of a small reflective ball at the instrument tip is accomplished using two position-sensitive detectors. The system is presently accurate to approximately $7 \mu \mathrm{m}$. Development is ongoing toward the goal of $1 \mu \mathrm{m}$ accuracy. Preliminary results have been presented. 


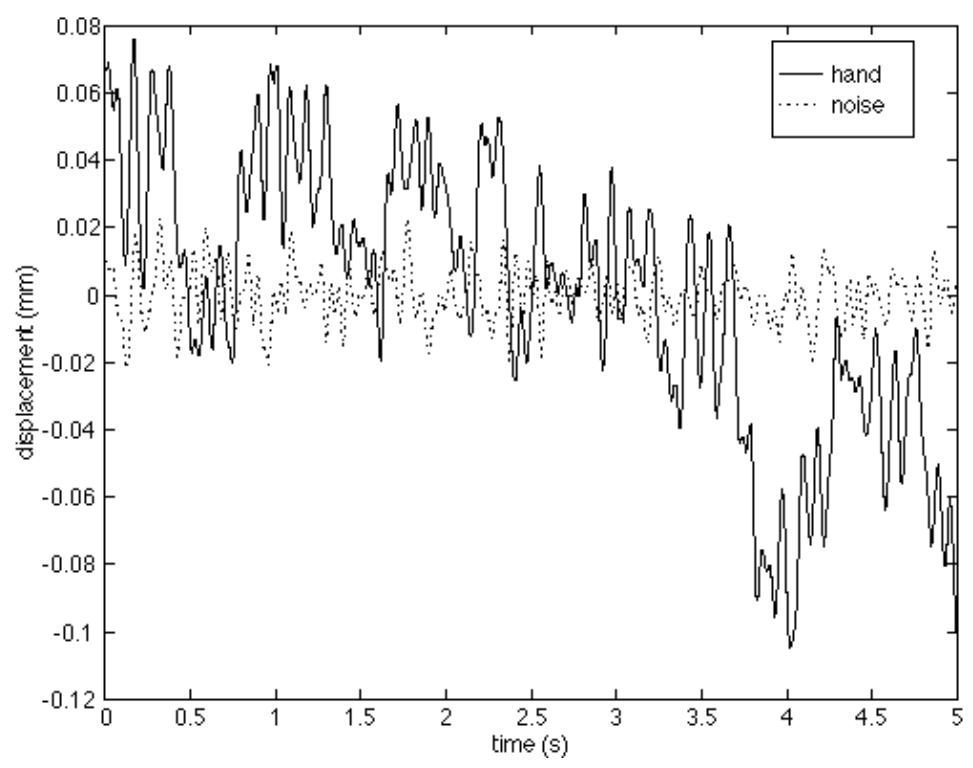

Fig. 5. Sample of data recorded while subject attempted to hold instrument motionless. The solid line depicts the motion in $x$, a horizontal coordinate. Both physiological tremor and lower-frequency aperiodic erroneous motion are visible. The dotted line presents, for comparison, a sample of the system noise in $x$ taken from a separate recording made moments earlier.

\section{Acknowledgments}

Funding is provided by the Johnson \& Johnson Focused Giving Program. The authors are grateful to Mr. C. Wen for software programming, Drs. M. Siegel and V. Brajovic for advice, Dr. R. Hollis for the use of equipment, and Mr. Jerry Lim for construction of the mannequin.

\section{References}

1. Patkin, M.: Ergonomics applied to the practice of microsurgery. Austr. N. Z. J. Surg. 47 (1977) 320-239. 1080

2. Riviere, C. N., Rader, R. S., Khosla, P. K.: Characteristics of hand motion of eye surgeons. Proc. 19th Annu. Conf. IEEE Eng. Med. Biol. Soc., Chicago (1997). 1080

3. Charles, S.: Dexterity enhancement for surgery. In: Taylor, R. H., Lavallée, S., Burdea, G. C., Mösges, R. (eds.): Computer Integrated Surgery: Technology and Clinical Applications. MIT Press, Cambridge (1996) 467-471. 1080

4. Hunter, I. W., Doukoglou, T. D., Lafontaine, S. R., Charette, P. G., Jones, L. A., Sagar, M. A., Mallinson, G. D., Hunter, P. J.: A teleoperated microsurgical robot and associated virtual environment for eye surgery. Presence 2 (1993) 265-280. 1080 
5. Schenker, P. S., Barlow, E. C., Boswell, C. D., Das, H., Lee, S., Ohm, T. R., Paljug, E. D., Rodriguez, G., Charles, S. T.: Development of a telemanipulator for dexterity enhanced microsurgery. Proc. 2nd Intl. Symp. Med. Robot. Comput. Assist. Surg. Wiley, New York (1995) 81-88. 1080

6. Salcudean, S. E., Ku, S., Bell, G.: Performance measurement in scaled teleoperation for microsurgery. In: Troccaz, J., Grimson, E., Mösges, R. (eds.): CVRMedMRCAS'97. Lecture Notes in Computer Science, Vol. 1205. Springer-Verlag, Berlin Heidelberg New York (1997) 789-798. 1080

7. Mitsuishi, M., Watanabe, H., Nakanishi, H., Kubota, H.: Dexterity enhancement for a tele-micro-surgery system with multiple macro-micro co-located operation point manipulators and understanding of the operator's intention. In: Troccaz, J., Grimson, E., Mösges, R. (eds.): CVRMed-MRCAS'97. Lecture Notes in Computer Science, Vol. 1205. Springer-Verlag, Berlin Heidelberg New York (1997) 821-830. 1080

8. Riviere, C. N., Rader, R. S., Thakor, N. V.: Adaptive canceling of physiological tremor for improved precision in microsurgery. IEEE Trans. Biomed. Eng. 45 (1998) 839-846. 1080

9. Simon, D. A. Intra-operative position sensing and tracking devices. In: Troccaz, J., Grimson, E., Mösges, R. (eds.): CVRMed-MRCAS'97. Lecture Notes in Computer Science, Vol. 1205. Springer-Verlag, Berlin Heidelberg New York (1997) 62-64. 1081

10. Simon, D. A., Jaramaz, B., Blackwell, M., Morgan, F., DiGioia, A. M., Kischell, E., Colgan, B., Kanade, T.: Development and validation of a navigational guidance system for acetabular implant placement. In: Troccaz, J., Grimson, E., Mösges, R. (eds.): CVRMed-MRCAS'97. Lecture Notes in Computer Science, Vol. 1205. Springer-Verlag, Berlin Heidelberg New York (1997) 583-592. 1081

11. Tseng, C.-S., Chung, C.-W., Chen, H.-H., Wang, S.-S., Tseng, H.-M.: Development of a robotic navigation system for neurosurgery. In: Westwood, J. D., Hoffman, H. E., Robb, R. A., Stredney, D. (eds.): Medicine Meets Virtual Reality. Studies in Health Technology and Informatics, Vol. 62. IOS Press, Amsterdam (1999) 358359. 1081

12. Sherman, K. P., Ward, J. W., Wills, D. P. M., Mohsen, A. M. M. A.: A portable virtual environment knee arthroscopy training system with objective scoring. In: Westwood, J. D., Hoffman, H. E., Robb, R. A., Stredney, D. (eds.): Medicine Meets Virtual Reality. Studies in Health Technology and Informatics, Vol. 62. IOS Press, Amsterdam (1999) 335-336. 1081

13. Rader, R. S., Walsh, A. C., Awh, C. C., de Juan, E., Jr.: Manual stability analysis of vitreoretinal microsurgery tasks. Ergonomics, in review. 1081

14. Schaefer, P., Williams, R. D., Davis, G. K., Ross, R. A.: Accuracy of position detection using a position-sensitive detector. IEEE Trans. Instrum. Meas. 47 (1998) 914-919. 1081, 1084, 1085

15. Ma, J.: Precision optical coordination sensor for cooperative 2-dof robots. M.S. report, Carnegie Mellon University (1998). 1081, 1084

16. Daghighian, H. M.: Optical position sensing with duolateral photoeffect diodes. Sensors (Nov. 1994) 35-40. 1082

17. Large-Area Position-Sensitive Detectors datasheet. Hamamatsu Corp., Bridgewater, N. J., USA (1988). 1082

18. Gomez-Blanco, M. A., Riviere, C. N., Khosla, P. K.: Intraoperative tremor monitoring for vitreoretinal microsurgery. Submitted to Medicine Meets Virtual Reality 8 (2000). 1085 